\title{
Perfume Manufacturing Microbes
}

\author{
Girish B Mahajan ${ }^{1 *}$ and Dipali Rahul Phatak ${ }^{2}$ \\ ${ }^{1}$ HiMedia laboratories Pvt. Ltd., Mumbai, India \\ ${ }^{2} J o g e s h w a r i$ Education Society, Mumbai, India \\ *Corresponding Author: Girish B Mahajan, HiMedia laboratories Pvt. Ltd., Mumbai, India.
}

Received: June 14, 2019; Published: July 08, 2019

DOI: 10.31080/ASMI.2019.02.0304

\begin{abstract}
Microbes (Actinomycetes, eubacteria, fungi, yeasts etc.) have displayed their potential to synthesize range of bioactive compounds, primary metabolites, enzymes, hormones etc. Synthesizing diverse range of perfume or flavour molecules is one more strong aspect of metabolism of microbes of certain genus. In the current article we presented this potential of microbes in an easy way. The topic is of high interest from innovative start-up point of view by initially exploring the right molecule.
\end{abstract}

Keywords: Yeast; Microbes; Plant

Whenever we encounter stink, invariably we think something is rotting, decomposing or of animal excreta etc. and indirectly blame microbes. Yes, most of the microbes we know are scavengers, degrading organic matter of plant or animal origin. Though smelly, decomposition of organic matter is an essential microbial process for recycling minerals and in balancing the elemental cycle in nature. Bacteria produce many odours, most of which that we'd rather not think about. The hundreds of volatile compounds that bacterial cultures produce can signal many things apart from imparting bad odour. When a microbe produces a volatile compound, it's a way of saying to its neighbouring microbe "hey listen to me.... It is also well established that much of our characteristic body smells are caused due to the metabolic activities of the prevalent type of microorganisms a person harbours. There is another aroma-charming side of microbes. If you want to appreciate some of the best perfume producers in nature, you will be astounded to know that they are none other than our tiny friends; the microbes.

Although "Perfume from Bacteria" sounds unconventional, they are the source for sweet, refreshing and pleasant fragrances. For ages, man has been using microbes to impart new aromas to fermentation products such as beer, wine, cheese etc. Let us begin with some of the most appealing perfumes in nature. After hot and sweaty summer when the first few drops of rain water touch the parched earth we experience the most divine scent of the earth; "Petrichor". This scent has enchanted several poets and writers to appreciate mother earth and express the romance of rainwater with the earth. Chemically this scent is known as Geosmin and is released when rain water falls on these microbes. The secret behind this essence is a microbe group called actinomycetes. Commercial production of this earthy scent is done in fermentation vessels using the special actinomycete.

Microorganisms produce the perfume molecules after their active phase of life. Recently, genetically engineered bacteria and yeasts are being developed that carry the perfume genes for producing fragrance from plants, like a surrogate mother. Biotechnology provides an attractive alternative for producing fragrant and flavour molecules in large quantities. Researchers used metabolic engineering to make E. coli produce a synthetic banana smell (Chemical name: Isoamyl acetate) and nicknamed it "Eau $d$ coli".

A rose by any other name would smell as sweet, because the scent is due to a type of alcohol called s 2-phenyl ethanol. The intoxicating scent of rose is well commercialised and produced by using strains of yeasts, such as Kluyveromyces marxianus, and Saccharomyces cerevisiae. They bring about bioconversion of 2-phenylalanine into 2-phenylethanol. 
When we close our eyes and relish yummy hot cheese in a burger or pizza, we inhale methyl ketones from the cheese. The wholesome aroma of cheese, is an appetite stimulant. The presence of methyl ketones was first observed in the spores of Penicillium roqueforti in a mould ripened cheese. The fungus converts fatty acid chains in cheese, with less than 14 carbon atoms into methyl ketones, making us crazy for cheese burgers and pizzas. The fragrance is the driving force of pizza houses and burger eateries.

Butane-2,3-dione (Diacetyl) is a naturally occurring flavour compound that possesses a strong typical 'butter' odour. The refreshing smell of butter reminds us of the famous Indian cuisine and Mumbai devised delicacy called 'Pav Bhaji'. Diacetyls are also used in perfumes and in reconstituting essential oils. The major diacetyl producing dairy bacteria are Lactococcus lactis, Lactobacillus sp, and Streptococcus thermophilus. The secret of successful dairy industries is to maintain the exact required panel of these dairy bacteria in optimal quantity in butter, curd and flavoured yogurts etc.

Coconut flakes, shreds, oil, cream are extensively used as food ingredients. Coconut aroma is very popular and chemically they include gamma-octalactone and gamma-nonalactone. Another lactone having a coconut odour is 6-pentyl-2-pyrone that is produced by fungus Trichoderma viridae. Some yeasts such as Candida tropicalis or Yarrowia lipolytica possess the ability to degrade ricinoleic acid and accumulate d-decalactone which contributes to fruity and oily flavour commonly found in peach, apricot and strawberry aromas. Without using coconut, the food can be given a feel of its presence by using such fermentation manufactured scents.

A class of organic compounds called esters contribute a fruity aroma to food. Esters such as ethyl acetate, hexyl acetate and 2-phenylethyl acetate provide typical flavour in wine and other grape-derived alcoholic beverages. The bacterium Lactococcus lactis possess unique esterase enzyme that is responsible for formation of such aroma ester compounds. The yeasts Hanseniaspora guilliermondii and Pichia anomala are potent 2-phenylethyl acetate and isoamyl acetate producers, respectively. Extraction of vanillin from vanilla plant beans is expensive and limited. But don't worry; microbes are there to control the cost of your vanilla ice-cream cone or cup. Several bacterial and fungal strains such as
Pseudomonas putida, and Aspergillus niger, are proficient in conversion of natural eugenol and isoeugenol from essential oils into vanillin. By now you would have realised that the sweet and tempting aroma of majority of our daily food is an outcome of our tiny and friendly microbes.

Luscious cherry aroma which is due to benzaldehyde is extracted from apricots. This extraction also leads to formation of toxic hydrocyanic acid that is undesirable. The only solution to this problem is to use the sweet microbes. Pseudomonas putida and the white rot fungi Trametes suaveolens, and Phanerochaete chrysosporium can be used as benzaldehyde producers.

Chocolate flavour is all-time treat for majority of kids. Toffees, cookies, biscuits, health drinks and even medicines for kids are chocolate flavoured. Thaumatin and monellin are the flavour active compounds. A type of yeast called Kluyveromyces sp. can produce this chocolate flavour, which is safe and can be scaled up to any required extent by using suitable reactors.

One example of the importance of our perfume producing tiny friends is worth mentioning. In 2010, there was a scarcity of patchouli oil, a fragrance used in incense and personal and home care products. Rainy weather in Indonesia caused a poor harvest of the shrub that produces the oil, and volcanic eruptions and earthquakes aggravated supply problems. Biotech firms like Allylix, Isobionics and Evolva came out with solution in the form of genetically engineering bacteria (e.g. Pseudomonas sp.) and yeast (Saccharomyces sp.) that can produce plant oils by fermenting sugars.

At present there are several such potential microbes which produce perfumes, which are not only a substitute source for plant and synthetic perfumes but also natural and scalable resources. The good part of microbes as perfume source is that their genes can be easily altered to get the distinct note of a particular aroma. Unlike plants, their growth is much faster. We expect more lovely, pleasant and sweet perfumes from microbes in future. The super diverse, tiny but mega-intelligence of microbes is yet to be explored for perfumery. Soon the probiotics formulae will be followed by "Aromabiotics" brands. Even in the perfumery area, microbes indicate "Don't underestimate the power of our aroma-metabolism". 


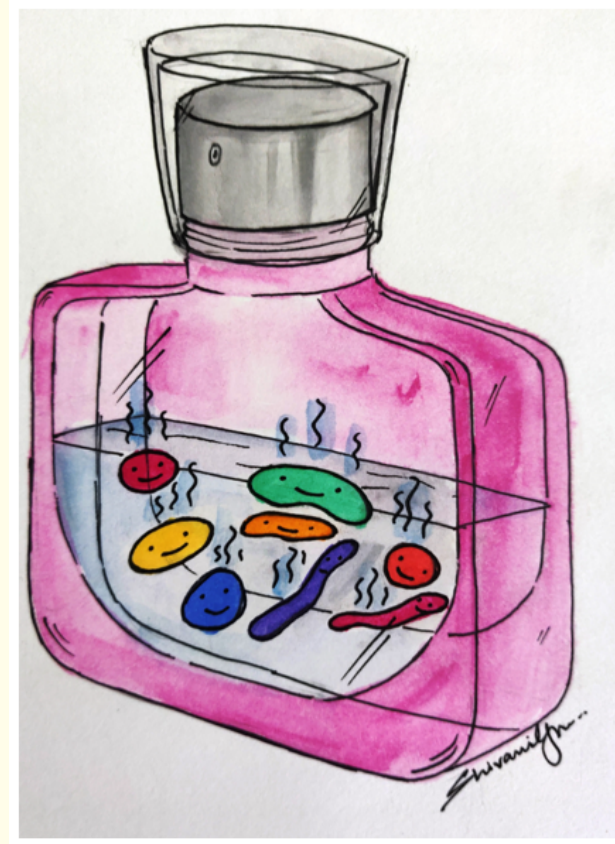

\section{Acknowledgment}

The authors are very thankful to their respective affiliation institutes for encouraging publishing of such awareness articles for wide readers of the journal. Authors of the article hereby gratefully acknowledge the Editor and Senior management of the monthly periodical titled 'Abhineet' published by 'Asha Foundation (https://indiaasha.org/)' for approving the copyrights for the publication of the article, which was published in 'Abhineet' in May 2019 issue. The authors are also thankful to Shivani G. Mahajan for drawing, painting and providing the apt and creative painting for this article.

Volume 2 Issue 8 August 2019

(C) All rights are reserved by Girish B Mahajan and Dipali Rahul Phatak. 